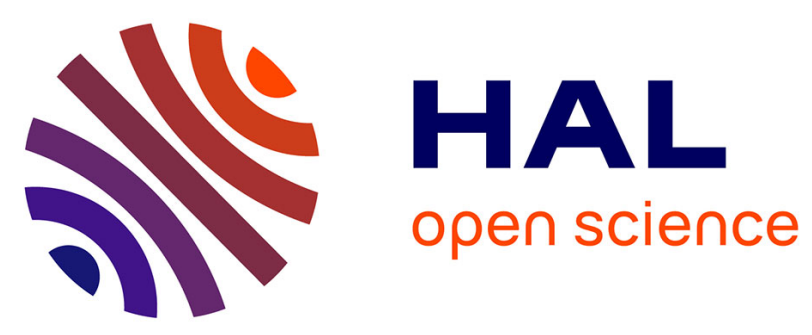

\title{
Atlas-Based Quantification of Myocardial Motion Abnormalities: Added-Value for Understanding the Effect of Cardiac Resynchronization Therapy
}

Nicolas Duchateau, Adelina Doltra, Etelvino Silva, Mathieu de Craene, Gemma Piella, Maria Angeles Castel, Lluis Mont, Josep Brugada, Alejandro Frangi, Marta Sitges

\section{To cite this version:}

Nicolas Duchateau, Adelina Doltra, Etelvino Silva, Mathieu de Craene, Gemma Piella, et al.. AtlasBased Quantification of Myocardial Motion Abnormalities: Added-Value for Understanding the Effect of Cardiac Resynchronization Therapy. Ultrasound in Medicine \& Biology, 2012, 38 (12), pp.21862197. 10.1016/j.ultrasmedbio.2012.08.009 . hal-02282422

\section{HAL Id: hal-02282422 \\ https://hal.science/hal-02282422}

Submitted on 7 Oct 2019

HAL is a multi-disciplinary open access archive for the deposit and dissemination of scientific research documents, whether they are published or not. The documents may come from teaching and research institutions in France or abroad, or from public or private research centers.
L'archive ouverte pluridisciplinaire HAL, est destinée au dépôt et à la diffusion de documents scientifiques de niveau recherche, publiés ou non, émanant des établissements d'enseignement et de recherche français ou étrangers, des laboratoires publics ou privés. 


\title{
Atlas-based Quantification of Myocardial Motion Abnormalities: added-value for Understanding the effect of Cardiac Resynchronization Therapy
}

\author{
Nicolas Duchateau*, Adelina Doltra ${ }^{+}$, Etelvino Silva ${ }^{+}$, Mathieu De Craene ${ }^{*}$, Gemma Piella ${ }^{*}$, Maria Ángeles Castel ${ }^{+}$, \\ Lluís Mont $^{\dagger}$, Josep Brugada ${ }^{\dagger}$, Alejandro F. Frangi ${ }^{*} \ddagger$, Marta Sitges $^{\dagger \S}$ \\ * Center for Computational Imaging \& Simulation Technologies in Biomedicine - Universitat Pompeu Fabra and \\ Centro de Investigación Biomédica en Red en Bioingeniería, Biomateriales y Nanomedicina, Barcelona, Spain; \\ † Thorax Institute, Hospital Clínic, Institut d'Investigacions Biomèdiques August Pi i Sunyer, Universitat de \\ Barcelona, Spain; \\ * Department of Mechanical Engineering, University of Sheffield, Sheffield, UK; \\ $\S$ The two last authors share senior authorship \\ Corresponding author: \\ Nicolas Duchateau \\ Center for Computational Imaging \& Simulation Technologies in Biomedicine (CISTIB) \\ Information \& Communications Technologies Department, Universitat Pompeu Fabra, \\ c/ Roc Boronat 138, E08018 Barcelona, Spain \\ Tel.: +34 93542 1348; fax: +34 935422083 . \\ E-mail: n.duchateaucistib@gmail.com (N. Duchateau)
}




\begin{abstract}
Statistical atlases may help improving the analysis of cardiac wall-motion abnormalities. This study aims at demonstrating the clinical value of such a method to better understand the effect of cardiac resynchronization therapy (CRT). We compared an atlas of normal septal motion built using apical 4-chamber 2D-echocardiographic sequences from healthy volunteers, to 88 patients undergoing CRT at baseline and 12 months follow-up. Abnormal motion was quantified locally using a $p$-value based on a statistical distance to normality. Reduction $\geq 15 \%$ in left ventricle endsystolic volume defined CRT response. Responders showed significantly higher reduction of abnormalities ( $p \leq 0.001)$. Non-responders conserved abnormal septal motion at the end of the isovolumic contraction (IVC). A specific inward-outward motion of the septum during IVC predominated in responders, and was corrected at followup. The method is of interest to characterize patterns of mechanical dyssynchrony, and study the link between their evolution and CRT response.
\end{abstract}

\title{
Key words
}

Speckle tracking; Motion atlas; Quantitative analysis; LV dyssynchrony; Cardiac resynchronization therapy. 


\section{Introduction}

The role of cardiac resynchronization therapy (CRT) in improving both clinical condition and cardiac function of heart failure patients has been clearly demonstrated (Abraham et al. 2002). The recovery of a synchronous contraction is a first necessary step to guarantee such an improvement, and from a broader perspective, that the patient can respond to the therapy. The importance of understanding the complexity and variety of the etiologies of cardiac dyssynchrony has been recently highlighted (Parsai et al. 2009a; Bijnens et al. 2006; Lumens et al. 2012; Leenders et al. 2012a) as an explanation to the limitations of single measurements of mechanical dyssynchrony (Fornwalt et al. 2009; Fornwalt 2011).

Echocardiography (Mor-Avi et al. 2011) with tissue Doppler and speckle tracking has shown its potential for estimating myocardial motion and deformation locally, and therefore quantifying mechanical dyssynchrony on individuals. However, its capability for intra- and inter- population comparison is limited, the analysis being performed at the regional level (myocardial segments) at some specific instants of the cardiac cycle, due to the lack of a common spatiotemporal system of coordinates to perform the comparison at any spatiotemporal location. In contrast, in methods derived from statistical atlases (Toga et al. 2006; Young and Frangi 2009), the data from each subject of a given population (shape or information defined at each point of this shape) is synchronized to a common reference anatomy, which provides a multivariate representation of the local anatomical and functional features of this population. For example, a statistical atlas of the left ventricle (LV) can contain information about myocardial velocities, strain or fiber structure at any spatiotemporal location of a reference shape of the LV. Recently, an atlasbased quantification of myocardial motion abnormalities was proposed (Duchateau et al. 2011a), where the velocities of each studied patient were characterized according to their distance to normality.

In the present study, we aim at demonstrating the usefulness of this technique for the characterization of abnormal patterns of cardiac motion, specifically applied to the field of CRT. Accordingly, we aim at showing the value of the atlas approach to characterize patterns of abnormal septal motion and the relation between their evolution and CRT response in a large group of patients pre- and post- CRT. 


\section{Materials and methods}

\section{Patient population}

For the present study, data was collected from 21 healthy volunteers and 88 patients undergoing CRT implantation. The baseline characteristics for these subjects are summarized in Table 1 .

Data from the healthy volunteers (age $30 \pm 5$ years, 14 male) served as control group for the construction of an atlas of normal motion. Enrollment criteria were that they had no history of cardiac disease and a normal echocardiographic exam. All of them showed a QRS duration $<120 \mathrm{~ms}$, and their baseline characteristics matched the values found in the literature for a population of patients with normal cardiac function (Feigenbaum 1994; Sutherland et al. 2006). Differences in age with the set of CRT candidates may be a limitation of this study, and is discussed at the end of this paper. Additional justifications about this population were extensively described in Duchateau et al. (2011a). The use of 21 healthy volunteers was justified by computing the evolution of the motion abnormality index depending on the size of the atlas population, and leave-one-out was used to check the normality of the motion of healthy volunteers.

The 88 patients compared to the atlas (age $68 \pm 9$ years, 64 male) were patients treated with CRT based on current international clinical guidelines (Dickstein et al. 2010): left ventricular ejection fraction (LVEF) $<35 \%$, QRS duration $>120 \mathrm{~ms}$, and NYHA classification III-IV or NYHA II who covered less than 500 meters in the 6 minutes walking test.

The research complied with the Declaration of Helsinki and the study protocol was accepted by our local ethics committee. Written informed consent was obtained from all subjects.

\section{Definition of response}

Volume response was defined as a reduction $\geq 15 \%$ in the LV end-systolic volume (Bleeker et al. 2006). Patients who died or had heart transplantation during the study were also considered as non-responders. Clinical improvement was defined as an increase $\geq 10 \%$ in the 6 minutes walking test, or a NYHA functional class reduction $\geq 1$ point for patients unable to complete the 6 minutes walking test at baseline. 


\section{Echocardiographic acquisition}

An echocardiographic examination using a commercially available system (Vivid 7, GE Healthcare, Milwaukee, WI, USA) was performed in all patients at baseline and at 12 months follow-up after the implant. A standard zoomed-in 4-chamber view of the LV was acquired during breath-hold to minimize the influence of respiratory motion. All sequences were acquired by the same sonographer, who optimized settings to achieve similar image quality for all subjects. Machine settings (gain, time gain compensation, and compression) were adjusted for optimal visualization, including the use of harmonic imaging (GE Healthcare M4S probe, 1.7/3.4 MHz frequency). Physiological differences between subjects constrain the acquisition parameters, which therefore differed in terms of temporal resolution and image quality. Resolution was optimized during the acquisition of healthy subjects' sequences, and corresponded to an average frame rate of $60 \mathrm{fps}$ and a pixel size of $0.24 \times 0.24 \mathrm{~mm}^{2}$. The CRT candidates involved in this study had dilated hearts compared to the healthy volunteers, and therefore required the use of a broader US sector. The temporal resolution of these patients' sequences was lower due to this constraint (around half the frame rate). Their average pixel size was $0.25 \times 0.25 \mathrm{~mm}^{2}$. The influence of the temporal resolution on the accuracy of the abnormality maps was commented in Duchateau et al. (2011a).

LV volumes and ejection fraction were measured using the 2D Simpson's method (biplane). Mitral regurgitation was semi-quantitatively classified into four degrees according to the color flow jet area method. LV dyssynchrony was also evaluated by two techniques: (1) measuring differences in time-to-peak myocardial velocity between the septal and the lateral walls of the LV, derived from color tissue Doppler (TDI) 4-chamber views (Bax et al. 2005); (2) measuring the time difference between peak systolic strain of the anteroseptal and inferolateral LV walls (Delgado et al. 2008). TDI images were not acquired during the healthy volunteers' examination and therefore the TDI septal-to-lateral delay is not available for this population.

\section{Automatic quantification of myocardial motion abnormalities}

The atlas pipeline described in Duchateau et al. (2011a) was applied to the acquired 4-chamber views of the $\mathrm{LV}$, focusing on the septal region (Figure 1). Its pre-processing stages (extraction of myocardial velocities and atlas synchronization) are detailed in Annex. 
Myocardial velocities were computed from each sequence using a non-rigid image registration algorithm, and spatiotemporally aligned to a common reference anatomy, using ECG matching (time) and image registration (space). The importance of this alignment step is commented in the Results section. Average and covariance of myocardial velocities over the set of healthy volunteers encoded a representation of normal motion. Abnormality in local motion was computed through a statistical distance on velocities, between each individual and the atlas population (Mahalanobis distance). This computation returned a $p$-value at every location of the myocardial wall, low $p$-value indicating high degree of abnormality.

A convenient way of representing this information consists of color-coded maps, inspired from anatomical M-mode echocardiographic images, in which the septal wall has been unfold around its medial line and used as vertical dimension, time being used as horizontal axis (Figure 1). The color code for these maps encodes the $p$-value in a logarithmic scale, multiplied by the sign of the radial velocity, to distinguish between septal abnormal motion patterns (Figure 2 and Results section). Blue color represents highly abnormal inward motion of the septum, while red color represents highly abnormal outward motion. No abnormality is therefore represented by white color.

The implementation of the method is not currently commercially available. Its implementation within an open-source platform will be achieved in further work in order to widen its use in clinical practice. Note that the current version of the non-rigid registration algorithm of De Craene et al. (2012a) is now available online (De Craene and Piella 2012b).

\section{Statistical analysis}

Normal distribution of quantitative variables was assessed using the Kolmogorov-Smirnov test. Normally distributed quantitative variables were expressed as mean \pm standard deviation, and unpaired Student's $t$-test was used for inter-groups comparison, while paired Student's $t$-test was used for the comparison of paired data. When large deviations from the Gaussian distribution were noticed, the variable was expressed as median and (interquartile) range, and Mann-Whitney $U$-test was preferred for inter-groups comparison, while Wilcoxon signedrank test was used for the comparison of paired data. Categorical variables were expressed in percentage over the 
number of patients for which data was available, and Fisher's exact test was used for inter-groups comparison, while McNemar's test was used for the comparison of paired data. $p$-values below 0.05 were considered as statistically significant difference between the tested groups. All data were analyzed using the SPSS statistical package (version 15.0, SPSS, Inc., Chicago, IL, USA). 


\section{Results}

\section{Patterns of motion abnormality at baseline}

Figure 2 represents the motion abnormality maps of five different subjects at baseline, during systole, illustrating the variety of patterns of abnormal motion that may be observed along the septal wall: (A) intraventricular dyssynchrony, assessed by the presence of a fast inward / outward motion of the septum during the isovolumic contraction period, otherwise called septal flash (SF [Parsai et al. 2009a]). Variations in the amplitude of $\mathrm{SF}$ are associated to variations in the intensity of the blue- and red-colored abnormalities on the map (Figure $2 a$ and $2 b$ ). This pattern was present in 60 patients (68\%) of our study group; (B) inter-ventricular dyssynchrony, with late systolic outward motion (Figure 2c), or with inward motion only (Figure $2 d$ ), referenced in the literature as left-right interaction (LR [Parsai et al. 2009a; Bijnens et al. 2006]). In contrast, no abnormal pattern is observed on a healthy volunteer (Figure 2e).

Animated joint views of the echocardiographic sequences for these five subjects and their corresponding abnormality maps are available as online data supplement.

\section{Importance of the atlas spatiotemporal alignment}

Figure 3 compares the maps of abnormality obtained for one patient with SF and one healthy volunteer, without (left) and with (right) the spatiotemporal alignment step inherent to the atlas construction. On the SF patient, the lack of spatiotemporal alignment overestimates the abnormality and the duration of the abnormality pattern, which does not fit anymore within the isovolumic contraction period. This is visible on both the abnormality map and the radial velocity curve. On the healthy volunteer, the lack of spatiotemporal alignment induces large abnormalities at the end of systole (due to artifacts on the longitudinal velocity computation in this case), which would make the subject being considered a false positive.

\section{Response to CRT}


Fifty-three patients $(60 \%)$ showed significant reverse remodeling and were considered responders. The clinical condition improved in 72 patients (82\%), 46 of them being also volume responders. One patient had heart transplantation, and died during the study. A second patient died from heart failure without heart transplantation.

\section{Prediction of response from baseline data}

There were no statistically significant differences at baseline between responders and non-responders for all the parameters (Table 2), except for (1) the amount of mitral regurgitation, moderate-severe mitral regurgitation being more frequent in non-responders $(p=0.036)$, and (2) septal-to-lateral delay derived from tissue Doppler, responders having more dyssynchrony at baseline as measured by this parameter $(p=0.040)$.

Baseline motion abnormalities as quantified by our method were compared to conventional indexes of dyssynchrony (TDI septal-to-lateral and speckle tracking anteroseptal-to-inferolateral delays) through a ROC analysis against volume response. Area-under-curve (AUC) and the ROC $p$-value are summarized in Table 3. The highest AUCs were observed for systolic abnormalities at basal- and mid-inferoseptal levels $(0.68$ and $0.67 ; p=0.040$ and 0.050 , respectively), while TDI- and speckle tracking-based conventional indexes showed lower performance ( 0.65 and $0.60 ; p=0.030$ and $>0.05$ [non-significant], respectively). The ROC curves for these four measurements are displayed in Figure 4.

\section{Overall effects of CRT}

The follow-up characteristics for the set of CRT candidates (parameters defining clinical and volume responses) are compared to the baseline ones in Table 4. As expected, due to the definition of clinical and volume responses, significant changes were observed for the group of responders for all parameters, contrary to the group of non-responders.

\section{Evolution of motion abnormalities with CRT}

The population of responders showed lower abnormalities at follow-up, and higher reduction of abnormalities in comparison with baseline. Values for the evolution of motion abnormalities in each spatiotemporal 
region are displayed in Table 5, the highest reduction being observed for responders at basal- and mid-inferoseptal levels $(p \leq 0.001)$.

The spatiotemporal localization of the motion abnormalities at baseline and follow-up is visible in Figure 5, which represents the average abnormality map for the groups of responders and non-responders. Little abnormality in septal motion was observed at follow-up for the group of responders, while non-responders still presented a peak of abnormality with inward motion at the end of the isovolumic contraction period, predominant at mid-inferoseptal level, supporting the regional observations of Table 5.

The patterns visible at baseline also indicated that large SF predominated in the group of responders, and was corrected at follow-up, while small SF and LR predominated in the group of non-responders. 


\section{Discussion}

The results of this study demonstrate the usefulness of the proposed atlas-based quantification of myocardial motion abnormalities for CRT studies, highlighting (1) the relevance of statistical indexes that intrinsically embed the notion of "normality", to characterize patterns of mechanical dyssynchrony and their evolution with the therapy; and (2) the need for a spatiotemporal synchronization of the data to avoid bias in the inter-subject comparison.

\section{Abnormal patterns of septal motion and cardiac dyssynchrony}

Parsai et al. (2009a) proposed a classification of patients according to their pattern of mechanical dyssynchrony, and studied CRT response for each of these groups. Half of the patients had SF, which was associated to CRT response when the mechanism had been corrected at follow-up. Similar observations were made on patients with contractile reserve, for which SF was induced by dobutamine stress echocardiography (Parsai et al. 2009b). The presence of an abnormal motion of the septum associated to an intra-ventricular type of dyssynchrony was first reported from M-mode observations (McDonald 1973; Dillon et al. 1974; Little et al. 1982; Grines et al. 1989). The authors described a specific pattern of mechanical dyssynchrony interpretable as a direct consequence of Left Bundle-Branch Block (LBBB [Parsai et al. 2009a; Bijnens et al. 2006; Duckett et al. 2012]). Gjesdal et al. (2011) interpreted it as the result of active septal contraction during LBBB and therefore suggested its inclusion in LV dyssynchrony studies. High response rates were recently reported for septal rebound stretch (De Boeck et al. 2009; Leenders et al. 2012b) and apical rocking (Szulik et al. 2010), two patterns very closely related to SF. Some elements of interpretation using a computer model were given in Lumens et al. (2012) and Leenders et al. (2012a). Interventricular dyssynchrony (LR) was described as a passive motion of the septum (Parsai et al. 2009a; Bijnens et al. 2006) due to the presence of infarcted septal regions and a long inter-ventricular delay, the correction of which led to clinical improvement but not volume response. Similar observations were made in Lumens et al. (2012) and Leenders et al. (2012a). More complex patterns were also reported, resulting from prolonged atrio-ventricular delays or combined mechanisms (Parsai et al. 2009a).

The objective of the present study was to consider all types of septal motion abnormalities that may be observed on our study group (Figure 2). This was achieved through the comparison of the motion of any individual 
to a control group with normal cardiac function, using a common system of coordinates to perform this comparison. This framework also allowed intra-subject comparison between baseline and follow-up data (Figure 5 and Table 5), to quantify the evolution of these patterns with the therapy.

\section{Conventional methods for dyssynchrony assessment}

The limits of single measurements from current echocardiographic techniques (Mor-Avi et al. 2011) such as tissue Doppler and speckle tracking have been largely discussed (Fornwalt et al. 2009; Fornwalt 2011). In particular, the relevance of considering individual components of the measured parameters (either temporally using time-topeak or time-to-onset measurements, or spatially by considering segmental values) has been pointed out. The practical difficulties of identifying the peak values (Anderson et al. 2008) may bias the analysis, despite the fact that statistical difference may be observed between responders and non-responders using these parameters (Table 2), and significant ROC $p$-values and AUCs (Figure 4 and Table 3). More elaborated methods have been proposed for analyzing this information (cross-correlation [Silva et al. 2010] or Fourier analysis [Bertola et al. 2009] of strain curves), but the complexity of the patterns of dyssynchrony may similarly limit their applicability.

To overcome these limitations, we chose a mechanistic approach inspired from the protocol presented in Parsai et al. (2009a), therefore considering the whole patterns of dyssynchrony that may be observed along the septum (Figure 2).

\section{Added-value of atlas-based quantification of motion abnormalities}

The protocol described in Parsai et al. (2009a) allows quantitative assessment of the abnormal patterns of septal motion (presence, timing and maximal excursion, if measurable), but is not automatic, pattern-dependent, and requires expert interpretation. Current echocardiographic techniques (Mor-Avi et al. 2011) such as tissue Doppler and speckle tracking allow the quantification of motion and deformation locally, but are still not adapted to perform intra- and inter-subject comparisons. Tissue Doppler is highly dependent on the insonation angle and only provides a one-dimensional measurement (the projection of the velocity vector along the observation beam), which limits its interest for our application. Both techniques process the sequence of each patient individually, but do not allow any 
comparison at any spatiotemporal location of a common system of coordinates. The need for such a comparison space, guaranteed by the use of a statistical atlas framework, was illustrated in Figure 3.

One of the main contributions of the present work consists in the computation of statistical indexes that intrinsically perform a comparison to normality. This concept is analogue to the learning process made by a clinical observer, which uses healthy and pathological sequences to learn the representations of specific patterns of abnormal motion. In our case, the analysis is completely quantitative, which allows baseline and follow-up comparisons, either regionally (Table 5) or locally at every point of the septal wall (Figure 5).

Our method slightly outperformed conventional methods for dyssynchrony assessment for the analysis of baseline data, as assessed by the ROC analysis presented in Figure 4 and Table 3. However, to our opinion, quantification and understanding remain more important than setting a single threshold for detection. Thus, our technique also allows pattern observation and analysis (as performed here, or using more advanced pattern-based comparison techniques as recently proposed [Duchateau et al. 2011b; Duchateau et al. 2012]), which is not possible with single indexes of dyssynchrony.

\section{Changes in LV dyssynchrony induced by CRT}

The primary objective of CRT is to restore the coordination in the motion of the cardiac chambers. With optimized resynchronization, cardiac function is expected to improve, leading to notable improvements in patient condition and allowing reverse remodeling of the LV (St John Sutton et al. 2003). The link between mechanical dyssynchrony and CRT response is still a controversial issue, partially due to the limitations of single measurements of mechanical dyssynchrony (Fornwalt et al. 2009; Fornwalt 2011). In contrast, mechanistic approaches (Parsai et al. 2009a; Bijnens et al. 2006; Lumens et al. 2012; Leenders et al. 2012a) do not discard the complexity of the etiologies of cardiac dyssynchrony and shed new light on the understanding of the effects of CRT. The therapy is expected to be highly efficient on patients with SF or septal rebound stretch, mechanical dyssynchrony being hypothesized to be mainly the consequence of an electrical problem (LBBB [Bijnens et al. 2006; Duckett et al. 2012; Gjesdal et al. 2011; Lumens et al. 2012; Leenders et al. 2012a]). In contrast, the therapy may have lower effect in case of passive motion of the septum (LR) or more complex mechanisms where mechanical dyssynchrony is a consequence of wall 
necrosis and akynesia of a given LV segment, rather unable to be corrected through electrical resynchronization (Parsai et al. 2009a; Lumens et al. 2012; Leenders et al. 2012a; Leenders et al. 2012b). Additional factors may also condition the ability of a patient to respond despite the reduction of the abnormal dyssynchrony patterns present at baseline. The presence of an extensive scar can limit the ability of the LV to show reverse remodeling in this region. The lack of contractile reserve (Lim et al. 2007), the presence of atrial fibrillation (Tolosana et al. 2008), an inappropriate lead position (Cowburn and Leclercq 2011), and the patient condition at baseline (too advanced heart failure so that the patient could not respond [Vidal et al. 2010]) may also strongly influence CRT response. The inclusion of additional variables of non-response such as the ones identified above is highly recommendable, and will lead to refinements of current multi-parametric analysis of CRT response (Lafitte et al. 2009; Santaularia-Tomas and Abraham 2009).

\section{Other potential clinical applications of statistical atlases of motion to cardiac imaging}

We chose to work with 2D echocardiography as it is the most widespread modality in clinical practice with sufficient temporal resolution to accurately identify fast motion patterns such as SF. Using 3D echocardiography would allow the characterization of all myocardial segments and the whole $3 \mathrm{D}$ motion of the heart, unlike $2 \mathrm{D}$ sequences, which consist of its projection on the observation plane. However, although 3D acquisitions are increasingly performed, the acquired sequences still have lower quality and temporal resolution than the $2 \mathrm{D}$ ones, which limits their ability to accurately quantify fast motion patterns.

We considered that apical 4-chamber represented the most relevant 2D view for our application, as we focus on types of mechanical dyssynchrony that may affect the whole septum, from base to apex. A reproducible position of the observation plane is harder to achieve in short axis views, which may be a strong limitation for building a statistical atlas from this view. Nonetheless, the methodology applied in this study is not specific to 2D echocardiography and abnormal septal motion, and could be readily applicable to other imaging modalities (3D echocardiography, magnetic resonance, as recently proposed by De Craene et al. (2012c), computed tomography, etc.), other clinical settings (stress echocardiography, ischemic cardiomyopathy, etc.), and other mechanisms of abnormal motion. 
The proposed technique could also be extended to compare individuals to a population with one of these specific abnormal motion patterns, and not only to normality, as described elsewhere (Duchateau et al. 2011b; Duchateau et al. 2012).

\section{Limitations}

The study focuses on motion abnormalities only. Computing maps of strain abnormalities would certainly help refining the analysis for patients who may have similar motion abnormality patterns despite different mechanisms. In particular, this may improve the understanding of CRT response, as the presence of local infarction may affect both the dyssynchrony patterns and the ability of the LV to remodel, and therefore, condition CRT response.

The atlas of normal motion was built from a group of healthy volunteers recruited for research purposes, within our institution, which explains the difference in age with the set of CRT candidates, and may be a limitation to our study. Recruitment of older volunteers was not performed for practical reasons. Nonetheless, the definition of a normal cardiac function still remains an open question. Cardiac efficiency may not be preserved when the subject gets older. Decreased myocardial velocities (mainly longitudinal component and diastolic period) were observed in older subjects (Sutherland et al. 2006; Wandt et al. 1998; Onose et al. 1999), resulting in a less "normal" function. Thus, the use of a young healthy population may be an advantage to perform comparison to a cardiac function as normal as possible. In particular, the quantification of motion abnormalities may be less contrasted with an older healthy population.

The current study focused on observations of voxel-wise motion abnormalities and did not attempt to do any classification of patients based on the observed patterns of abnormal motion, which would require the use of specific pattern analysis techniques (Duchateau et al. 2011b). The relation between patterns of mechanical dyssynchrony and CRT response is still complex (Parsai et al. 2009a; Lumens et al. 2012; Leenders et al. 2012a) and such an analysis may also take into account external factors that condition CRT response, as commented previously. 
Finally, the criteria for CRT response should also be considered carefully (Fornwalt et al. 2010; Cleland and Ghio 2011). In particular, the definition of fixed thresholds for defining response is certainly a limit for a clear understanding of the effects of CRT, in comparison with the use of a spectrum of responses, involving additional measures of CRT outcome (Foley et al. 2009).

\section{Conclusions}

The results presented in this study demonstrated the usefulness of an atlas-based quantification of motion abnormalities for characterizing the evolution of specific patterns of dyssynchrony with CRT. The proposed abnormality maps showed that abnormalities reduced significantly more in responders $(p \leq 0.001)$, while abnormal motion was still present in non-responders. They also showed that a large septal flash pattern predominated at baseline in the group of responders, while small septal flash and left-right interaction patterns predominated in the group of non-responders. We also discussed the combination of the proposed technique with a comprehensive understanding of the etiologies of cardiac dyssynchrony, to achieve a better interpretation of the effects of the therapy. 


\section{Annex: atlas pre-processing stages}

This annex details the pre-processing stages of the atlas pipeline (Duchateau et al. 2011a) introduced in the Material and methods section.

\section{Extraction of myocardial velocities}

Myocardial velocities were extracted from each sequence, at any spatiotemporal location, using non-rigid image registration. Our implementation performs pairwise matching of consecutive frames in the sequence, using multiscale diffeomorphic free-form-deformation (Rueckert et al. 2006). Its recent improvements to include temporal consistency and extensive validation are described in De Craene et al. (2012a). The concept of the speckle tracking algorithm, widely used in the clinical community, is similar to the tracking achieved through image registration, with the specificity of computing image similarities locally (tracking local speckle pattern). Although few details are given by the manufacturer regarding its implementation (which is the reason why we opted for the free-formdeformation algorithm), we believe the algorithm to follow the principles given in (Behar et al. 2004; Adam et al. 2004; Leitman et al. 2004). The intra-sequence tracking quality was evaluated for both volunteers and CRT candidates in Duchateau et al. (2011a), and showed a precision comparable to the variability of experienced observers in performing manual tracking.

\section{Atlas synchronization}

The velocities extracted from each sequence were then spatiotemporally aligned to a common reference anatomy, chosen among the set of healthy volunteers using the group-wise normalized mutual information metric (Hoogendoorn et al. 2010). Temporal alignment was based on piecewise linear warping of the timescale based on the timing of physiological events (onset of QRS complex [beginning and end of the cycle], located on the ECG using tools from the EchoPac software [GE Healthcare, Milwaukee, WI, USA], and the aortic valve opening and closure, determined using continuous wave Doppler imaging on the aortic valve). The reproducibility of both the echocardiographic acquisition and the atlas pipeline was evaluated in Duchateau et al. (2011a), through the quantification of the variability in the myocardial velocities of four different sequences of the same volunteer, once these have been spatiotemporally aligned. Spatial normalization consisted in estimating a mapping between the anatomy of the studied subject and the reference anatomy (multiscale diffeomorphic free-form-deformation 
[Rueckert et al. 2006], as for the intra-sequence registration), and locally reorienting the myocardial velocities using this mapping. 


\section{Acknowledgements}

\section{Funding}

This work was supported by the Spanish Industrial and Technological Development Center (cvREMOD CEN20091044), the Spanish Ministry of Science and Innovation, Plan E and the European Regional Development Fund (STIMATH TIN2009-14536-C02-01), the European Commission's Seventh Framework Program (EuHeart FP7-ICT224495), and the Spanish Health Institute Carlos III (REDINSCOR RD06/0003/0008). AD was supported by a PostResidency Award from Fundació Clínic. The authors gratefully acknowledge the support and advice for the atlas construction and the data collection of Prof. BH Bijnens from the Universitat Pompeu Fabra and the Institució Catalana de Recerca i Estudis Avançats, Barcelona, Spain.

\section{Conflict of interest}

None. 


\section{References}

Abraham WT, Fisher WG, Smith AL, Delurgio DB, Leon AR, Loh E, Kocovic DZ, Packer M, Clavell AL, Hayes DL, Ellestad M, Messenger J, and the MIRACLE study group. Cardiac resynchronization in chronic heart failure. N Engl J Med 2002;346:1845-53.

Adam D, Landesberg A, Konyukhov E, Lysyansky P, Lichtenstein O, Smirin N, Friedman Z. On changing coordinate systems for longitudinal tensor-based morphometry. In: Proceedings of IEEE Computers in Cardiology 2004. pp.337-40.

Anderson LJ, Miyazaki C, Sutherland GR, Oh JK. Patient selection and echocardiographic assessment of dyssynchrony in cardiac resynchronization therapy. Circulation 2008;117:2009-23.

Bax J, Abraham JT, Barold SS, Breithardt OA, Fung JWH, Garrigue S, Gorcsan III J, Hayes DL, Kass DA, Knuuti J, Leclercq C, Linde C, Mark DB, Monaghan MJ, Nihoyannopoulos P, Schalij MJ, Stellbrink C, and Yu CM. Cardiac resynchronization therapy: Part 1-issues before device implantation. J Am Coll Cardiol 2005;46:2153-67.

Behar V, Adam D, Lysyansky P, Friedman Z. The combined effect of nonlinear filtration and window size on the accuracy of tissue displacement estimation using detected echo signals. Ultrasonics 2004 ;41:743-53.

Bertola B, Rondano E, Sulis M, Sarasso G, Piccinino C, Marti G, Devecchi P, Magnani A, Francalacci G, Marino PN. Cardiac Dyssynchrony Quantitated by Time-to-Peak or Temporal Uniformity of Strain at Longitudinal, Circumferential, and Radial Level: Implications for Resynchronization Therapy. J Am Soc Echocardiogr 2009;22:665-71.

Bijnens B, Claus P, Parsai C, Weidemann F, Marciniak A, Anderson L, Sutherland GR. Assessing cardiac function in dilated and failing hearts. In: Sutherland GR, Hatle L, Claus P, D’hooge J, Bijnens B, eds. Doppler Myocardial Imaging, a textbook. BSWK-Hasselt, Belgium, 2006. pp. 251-77.

Bleeker GB, Bax JJ, Fung JWH, van der Wall EE, Zhang Q, Schalij MJ, Chan JYS, Yu CM. Clinical versus echocardiographic parameters to assess response to cardiac resynchronization therapy. Am J Cardiol 2006;97:260-3.

Cleland JG, Ghio S. The determinants of clinical outcome and clinical response to CRT are not the same. Heart Fail Rev 2011 (in press). 
Cowburn PJ, Leclercq C. How to improve outcomes with cardiac resynchronisation therapy: importance of lead positioning. Heart Fail Rev 2011 (in press).

De Boeck BW, Teske AJ, Meine M, Leenders GE, Cramer MJ, Prinzen FW, Doevendans PA. Septal rebound stretch reflects the functional substrate to cardiac resynchronization therapy and predicts volumetric and neurohormonal response. Eur J Heart Fail 2009;11:863-71.

De Craene M, Piella G, Camara O, Duchateau N, Silva E, Doltra A, D’hooge J, Brugada J, Sitges M, Frangi AF. Spatiotemporal diffeomorphic free-form deformation: application to motion and strain estimation from 3D echocardiography. Med Image Anal 2012;16(2):427-50.

De Craene M, Piella G. An implementation of the TDFFD and LDFFD algorithms. Insight Journal 2012. http://hdl.handle.net/10380/3345

De Craene M, Duchateau N, Tobon-Gomez C, Ghafaryasl B, Piella G, Rhode KS, Frangi AF. SPM to the heart: Mapping of 4D continuous velocities for motion abnormality quantification. In: Proceedings of IEEE International Symposium on Biomedical Imaging, 2012. pp. 454-7.

Delgado V, Ypenburg C, van Bommel RJ, Tops LF, Mollema SA, Marsan NA, Bleeker GB, Schalij MJ, Bax JJ. Assessment of left ventricular dyssynchrony by speckle tracking strain imaging comparison between longitudinal, circumferential, and radial strain in cardiac resynchronization therapy. J Am Coll Cardiol 2008;51:1944-52.

Dickstein K, Vardas PE, Auricchio A, Daubert JC, Linde C, McMurray J, Ponikowski P, Priori SG, Sutton R, van Veldhuisen DJ. 2010 Focused update of ESC guidelines on device therapy in heart failure: an update of the 2008 ESC guidelines for the diagnosis and treatment of acute and chronic heart failure and the 2007 ESC guidelines for cardiac and resynchronization therapy. Eur Heart J 2010;31:2677-87.

Dillon JC, Chang S, Feigenbaum.H. Echocardiographic manifestations of left-bundle branch-Block. Circulation 1974;49:876-80.

Duchateau N, De Craene M, Piella G, Silva E, Doltra A, Sitges M, Frangi AF. A spatiotemporal Statistical atlas of motion for the quantification of abnormal myocardial tissue velocities. Med Image Anal 2011;15:316-28.

Duchateau N, De Craene M, Piella G, Frangi AF. Characterizing Pathological Deviations from Normality using Constrained Manifold-Learning. In: Fichtinger G, Martel A, Peters T, eds. Proceedings of Med Image Comput Comput Assist Interv. Springer LNCS, 2011. vol. 6893, pp. 256-63. 
Duchateau N, De Craene M, Piella G, Frangi AF. Constrained Manifold Learning for the Characterization of Pathological Deviations from Normality. Med Image Anal 2012 (in press).

Duckett SG, Camara O, Ginks MR, Bostock J, Chinchapatnam P, Sermesant M, Pashaei A, Lambiase PD, Gill JS, Carr-White GS, Frangi AF, Razavi R, Bijnens BH, Rinaldi CA. Relationship between endocardial activation sequences defined by high-density mapping to early septal contraction (septal flash) in patients with left bundle branch block undergoing cardiac resynchronization therapy. Europace, 2012;14:99-106.

Feigenbaum H. Echocardiographic measurements and normal values. In: Feigenbaum H, ed. Echocardiography. Philadelphia: Lea and Febiger, 1994. pp. 658-95.

Foley PWX, Leyva F, Frenneaux MP. What is treatment success in cardiac resynchronization therapy? Europace 2009;11:v58-65.

Fornwalt BK, Delfino JG, Sprague WW, Oshinski JN. It's time for a paradigm shift in the quantitative evaluation of left ventricular dyssynchrony. J Am Soc Echocardiogr 2009;22:672-6.

Fornwalt BK, Sprague WW, BeDell P, Suever JD, Gerritse B, Merlino JD, Fyfe DA, León AR, Oshinski JN. Agreement is poor among current criteria used to define response to cardiac resynchronization therapy. Circulation 2010;121:1985-91.

Fornwalt BK. The dyssynchrony in predicting response to cardiac resynchronization therapy: a call for change. J Am Soc Echocardiogr 2011;24:180-4.

Gjesdal O, Remme EW, Opdahl A, Skulstad H, Russell K, Kongsgaard E, Edvardsen T, Smiseth OA. Mechanisms of abnormal systolic motion of the interventricular septum during left bundle-branch block. Circ Cardiovasc Imaging 2011;4:264-73.

Grines CL, Bashore TM, Boudoulas H, Olson S, Shafer P, Wooley CF. Functional abnormalities in isolated left bundle branch block. The effect of interventricular asynchrony. Circulation 1989;79:845-53.

Hoogendoorn C, Whitmarsh T, Duchateau N, Sukno FM, De Craene M, Frangi AF. A groupwise mutual information metric for cost efficient selection of a suitable reference in cardiac computational atlas construction. In: Proceedings of SPIE International Conference on Medical Imaging 2010. pp.76231R.

Lafitte S, Reant P, Zaroui A, Donal E, Mignot A, Bougted H, Belghiti H, Bordachar P, Deplagne A, Chabaneix J, Franceschi F, Deharo JC, Dos Santos P, Clementy J, Roudaut R, Leclercq C, Habib G. Validation of an 
echocardiographic multiparametric strategy to increase responders patients after cardiac resynchronization: a multicentre study. Eur Heart J 2009;30:2880-7.

Leenders GE, Lumens J, Cramer MJ, De Boeck BW, Doevendans PA, Delhaas T, Prinzen FW. Septal deformation patterns delineate mechanical dyssynchrony and regional differences in contractility: analysis of patient data using a computer model. Circ Heart Fail 2012;5:87-96.

Leenders GE, De Boeck BW, Teske AJ, Meine M, Bogaard MD, Prinzen FW, Doevendans PA, Cramer MJ. Septal rebound stretch is a strong predictor of outcome after cardiac resynchronization therapy. J Card Fail 2012;18:404-12.

Leitman M, Lysyansky P, Sidenko S, Shir V, Peleg E, Binenbaum M, Kaluski E, Krakover R, Vered Z. Twodimensional strain-a novel software for real-time quantitative echocardiographic assessment of myocardial function. J Am Soc Echocardiogr 2004;17:1021-9.

Lim P, Bars C, Mitchell-Heggs L, Roiron C, Elbaz N, Hamdaoui B, Lellouche N, Dubois-Randé JL, Guéret P. Importance of contractile reserve for CRT. Europace 2007;9:739-43.

Little WC, Reeves RC, Arciniegas J, Katholi RE, Rogers EW. Mechanism of abnormal inter-ventricular septal motion during delayed left-ventricular activation. Circulation 1982;65:1486-91.

Lumens J, Leenders GE, Cramer MJ, De Boeck BW, Doevendans PA, Prinzen FW, Delhaas T. Mechanistic evaluation of echocardiographic dyssynchrony indices: patient data combined with multiscale computer simulations. Circ Cardiovasc Imaging 2012;5:491-9.

McDonald IG. Echocardiographic demonstration of abnormal motion of the interventricular septum in left bundle branch block. Circulation 1973;48:272-80.

Mor-Avi V, Lang RM, Badano LP, Belohlavek M, Cardim NM, Derumeaux G, Galderisi M, Marwick T, Nagueh SF, Sengupta PP, Sicari R, Smiseth OA, Smulevitz B, Takeuchi M, Thomas JD, Vannan M, Voigt JU, Zamorano JL. Current and evolving echocardiographic techniques for the quantitative evaluation of cardiac mechanics: ASE/EAE consensus statement on methodology and indications endorsed by the Japanese Society of Echocardiography. Eur J Echocardiogr 2011;12:167-205.

Onose Y, Oki T, Mishiro Y, Yamada H, Abe M, Manabe K, Kageji Y, Tabata T, Wakatsuki T, Ito S. Influence of aging on systolic left ventricular wall motion velocities along the long and short axes in clinically normal patients determined by pulsed tissue Doppler imaging. J Am Soc Echocardiogr 1999;12:921-6. 
Parsai C, Bijnens B, Sutherland GR, Baltabaeva A, Claus P, Marciniak M, Paul V, Scheffer M, Donal E, Derumeaux G, Anderson L. Toward understanding response to cardiac resynchronization therapy: left ventricular dyssynchrony is only one of multiple mechanisms. Eur Heart J 2009;30:940-9.

Parsai C, Baltabaeva A, Anderson L, Chaparro M, Bijnens BH, Sutherland GR. Low-dose dobutamine stress echo to quantify the degree of remodelling after cardiac resynchronization therapy. Eur Heart J 2009;30:950-8.

Rueckert D, Aljabar P, Heckemann RA, Hajnal JV, Hammers A. Diffeomorphic registration using Bsplines. In: Larsen R, Nielsen M, Sporring J, eds. Proceedings of Med Image Comput Comput Assist Interv. Springer LNCS, 2006. vol. 4191, pp. 702-9.

Santaularia-Tomas M, Abraham TP. Criteria predicting response to CRT: is more better? Eur Heart J 2009;30:28357.

Silva E, Sitges M, Doltra A, Mont L, Vidal B, Castel MA, Tolosana JM, Berruezo A, González Juanatey JR, Brugada. Analysis of temporal delay in myocardial deformation throughout the cardiac cycle: Utility for selecting candidates for cardiac resynchronization therapy. Heart Rhythm 2010;7:1580-6.

St John Sutton MG, Plappert T, Abraham WT, Smith AL, DeLurgio DB, Leon AR, Loh E, Kocovic DZ, Fisher WG, Ellestad M, Messenger J, Kruger K, Hilpisch KE, Hill MRS, and for the Multicenter InSync Randomized Clinical Evaluation (MIRACLE) Study Group. Effect of cardiac resynchronization therapy on left ventricular size and function in chronic heart failure. Circulation 2003;107:1985-90.

Sutherland GR, Hatle L, Claus P, Herbots L, Šeparović J. Normal data. In: Sutherland GR, Hatle L, Claus P, D’hooge J, Bijnens B, eds. Doppler Myocardial Imaging, a textbook. BSWK-Hasselt, Belgium, 2006. pp.49-102.

Szulik M, Tillekaerts M, Vangeel V, Ganame J, Willems R, Lenarczyk R, Rademakers F, Kalarus Z, Kukulski T, Voigt JU. Assessment of apical rocking: a new, integrative approach for selection of candidates for cardiac resynchronization therapy. Eur J Echocardiogr 2010;11:863-9.

Toga AW, Thompson PM, Mori S, Amunts K, Zilles K. Towards multimodal atlases of the human brain. Nat Rev Neurosci 2006;7:952-66.

Tolosana JM, Hernandez Madrid A, Brugada J, Sitges M, Garcia Bolao I, Fernandez Lozano I, Ferrer JM, Quesada A, Macias A, Marin W, Escudier JM, Alonso Gomez A, Gimenez Alcala M, Tamborero D, Berruezo A, Mont L, and on behalf of the SPARE Investigators. Comparison of benefits and mortality in cardiac 
resynchronization therapy in patients with atrial fibrillation versus patients in sinus rhythm (Results of the Spanish Atrial Fibrillation and Resynchronization [SPARE] Study). Am J Cardiol 2008;102:444-9.

Vidal B, Delgado V, Mont L, Poyatos S, Silva E, Castel MA, Tolosana JM, Berruezo A, Brugada J, Sitges M. Decreased likelihood of response to cardiac resynchronization in patients with severe heart failure. Eur $\mathrm{J}$ Heart Fail 2010;12:283-7.

Wandt B, Bojö L, Hatle L, Wranne B. Left ventricular contraction pattern changes with age in normal adults. J Am Soc Echocardiogr 1998;11:857-93.

Young AA, Frangi AF. Computational cardiac atlases: from patient to population and back. Exp Physiol 2009;94:578-96. 
Table 1. Baseline characteristics of volunteers and CRT candidates.

\begin{tabular}{lcc}
\hline & CRT candidates (N=88) & Volunteers (N=21) \\
\hline Age (years) & $68 \pm 9$ & $30 \pm 5$ \\
Male gender & $64(73 \%)$ & $14(67 \%)$ \\
Ischemic etiology & $29(33 \%)$ & 0 \\
QRS width (ms) & $178 \pm 29$ & $81 \pm 10$ \\
6min walking test (m) & $271(168-332)$ & $\cdot$ \\
$\quad$ I & 0 & 0 \\
$\quad$ II & $23(26 \%)$ & 0 \\
$\quad$ III & $56(65 \%)$ & 0 \\
$\quad$ IV & $7(8 \%)$ & 0 \\
Z & $247 \pm 88$ & $104 \pm 27$ \\
LV end-diastolic volume (mL) & $186 \pm 76$ & $41 \pm 9$ \\
LV end-systolic volume (mL) & $25 \pm 8$ & $60 \pm 5$ \\
LV ejection fraction (\%) & $26(43 \%)$ & 0 \\
MR grade III-IV (\%) & $63 \pm 41$ & $\cdot$ \\
TDI septal-to-lateral delay (ms) & $66(28-158)$ & $17 \pm 27$ \\
ST anteroseptal-to-inferolateral delay (ms) &
\end{tabular}

LV: left ventricular; MR: mitral regurgitation; TDI: tissue Doppler imaging; ST: speckle tracking 
Table 2. Baseline characteristics of CRT candidates according to the volume response.

\begin{tabular}{|c|c|c|c|c|}
\hline \multicolumn{3}{|c|}{ Responders $(\mathrm{N}=53)$} & Non-responders $(\mathrm{N}=35)$ & $p$-value \\
\hline \multicolumn{2}{|c|}{ Age (years) } & $67 \pm 9$ & $69 \pm 8$ & NS \\
\hline \multicolumn{2}{|c|}{ Male gender } & $36(68 \%)$ & $28(80 \%)$ & NS \\
\hline \multicolumn{2}{|c|}{ Ischemic etiology } & $15(28 \%)$ & $14(31 \%)$ & NS \\
\hline \multicolumn{2}{|c|}{ QRS width (ms) } & $181 \pm 27$ & $172 \pm 31$ & NS \\
\hline \multicolumn{2}{|c|}{ 6min walking test $(\mathrm{m})$} & $275(197-320)$ & $270(0-336)$ & NS \\
\hline \multirow{4}{*}{ 乙 } & $\mathbf{I}$ & 0 & 0 & . \\
\hline & II & $14(27 \%)$ & $9(27 \%)$ & NS \\
\hline & III & $36(69 \%)$ & $20(59 \%)$ & NS \\
\hline & IV & $2(4 \%)$ & $5(15 \%)$ & NS \\
\hline \multicolumn{2}{|c|}{ LV end-diastolic volume (mL) } & $256 \pm 95$ & $231 \pm 75$ & NS \\
\hline \multicolumn{2}{|c|}{ LV end-systolic volume (mL) } & $196 \pm 79$ & $172 \pm 68$ & NS \\
\hline \multicolumn{2}{|c|}{ LV ejection fraction (\%) } & $25 \pm 7$ & $27 \pm 9$ & NS \\
\hline \multicolumn{2}{|c|}{ MR grade III-IV (\%) } & $10(29 \%)$ & $16(59 \%)$ & 0.036 \\
\hline \multicolumn{2}{|c|}{ TDI septal-to-lateral delay (ms) } & $70 \pm 36$ & $51 \pm 46$ & 0.040 \\
\hline \multicolumn{2}{|c|}{ ST anteroseptal-to-inferolateral delay (ms) } & $70(33-182)$ & $48(21-120)$ & NS \\
\hline
\end{tabular}

LV: left ventricular; MR: mitral regurgitation; TDI: tissue Doppler imaging; ST: speckle tracking

NS: Non-significant statistical difference ( $p$-value $>0.05)$. 
Table 3. ROC analysis against volume response of baseline motion abnormalities and conventional indexes of dyssynchrony.

\begin{tabular}{|c|c|c|c|}
\hline & & Area under curve & $p$-value \\
\hline \multirow{3}{*}{$\begin{array}{l}\frac{0}{0} \\
\frac{0}{\omega} \\
\text { के }\end{array}$} & $\overline{B I}$ & 0.68 & 0.040 \\
\hline & MI & 0.67 & 0.050 \\
\hline & AS & 0.56 & NS \\
\hline \multirow{3}{*}{$\begin{array}{l}\frac{0}{3} \\
\frac{9}{2} \\
\frac{\pi}{0}\end{array}$} & BI & 0.60 & NS \\
\hline & MI & 0.64 & NS \\
\hline & AS & 0.57 & NS \\
\hline \multicolumn{2}{|c|}{ TDI septal-to-lateral delay (ms) } & 0.65 & 0.030 \\
\hline \multicolumn{2}{|c|}{ ST anteroseptal-to-inferolateral delay (ms) } & 0.60 & NS \\
\hline
\end{tabular}

BI: basal-inferoseptal; MI: mid-inferoseptal; AS: apical septal; TDI: tissue Doppler imaging; ST: speckle tracking

NS: Non-significant statistical difference ( $p$-value $>0.05)$. 
Table 4. Comparison of follow-up and baseline characteristics (parameters defining clinical and volume response) of CRT candidates according to the volume response.

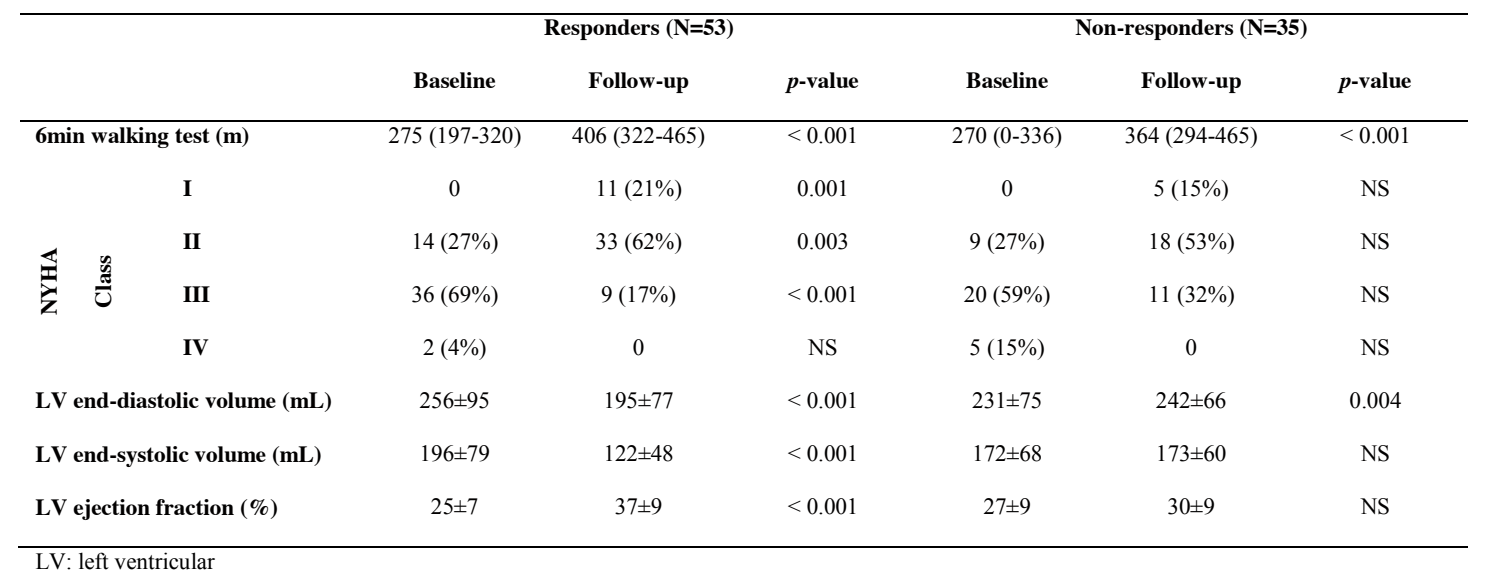

NS: Non-significant statistical difference ( $p$-value $>0.05)$. 
Table 5. Abnormality reduction according to the volume response.

\begin{tabular}{|c|c|c|c|c|c|c|c|}
\hline & & \multicolumn{3}{|c|}{ Responders (N=53) } & \multicolumn{3}{|c|}{ Non-responders ( $\mathbf{N = 3 5 )}$} \\
\hline & & Baseline & Follow-up & $p$-value & Baseline & Follow-up & $p$-value \\
\hline \multirow{3}{*}{$\begin{array}{l}\frac{0}{0} \\
\frac{0}{0} \\
\frac{0}{2}\end{array}$} & $\mathrm{BI}$ & $1.02(0.84-1.27)$ & $0.74(0.64-0.99)$ & $<0.001$ & $0.96(0.72-1.16)$ & $0.81(0.73-0.97)$ & NS \\
\hline & MI & $1.15(0.97-1.74)$ & $0.86(0.66-1.22)$ & 0.001 & $1.26(0.81-1.58)$ & $1.03(0.80-1.30)$ & NS \\
\hline & AS & $0.82(0.65-0.99)$ & $0.78(0.63-1.06)$ & NS & $0.68(0.54-1.10)$ & $0.78(0.66-0.99)$ & NS \\
\hline \multirow{3}{*}{ 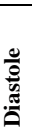 } & $\mathrm{BI}$ & $0.76(0.52-1.22)$ & $0.55(0.46-0.80)$ & 0.014 & $0.65(0.51-0.89)$ & $0.57(0.47-0.74)$ & NS \\
\hline & MI & $0.96(0.62-1.71)$ & $0.68(0.52-1.20)$ & 0.013 & $0.78(0.55-1.62)$ & $0.77(0.53-0.94)$ & 0.050 \\
\hline & AS & $0.90(0.62-1.23)$ & $0.66(0.51-1.03)$ & 0.030 & $0.84(0.46-1.45)$ & $0.70(0.51-1.04)$ & NS \\
\hline
\end{tabular}

$p$-value corresponds to the discrimination score between baseline and follow-up abnormalities.

BI: basal-inferoseptal; MI: mid-inferoseptal; AS: apical septal

NS: Non-significant statistical difference $(p$-value $>0.05)$ 


\section{Figure legends}

Figure 1: Pipeline for the atlas-based quantification of septal motion abnormalities, as described in Duchateau et al. (2011a). Right part represents the motion abnormality map computed for one patient with septal flash during systole ( $p$-value in logarithmic scale weighted by the sign of the radial velocity $\mathrm{v}_{\mathrm{p}}$ ). Red arrows indicate the localization of inward (IN) and outward (OUT) events of septal flash. The vertical line indicates the end of the isovolumic contraction period.

Figure 2: Variety of the patterns observed on the motion abnormality maps (systolic period).

Figure 3: Velocity profiles and motion abnormality maps for one patient and one healthy volunteer without and with the atlas spatiotemporal synchronization. Dashed line on the abnormality map indicate the septal level at which velocity profile are represented. Gray profile and errorbars indicate the average \pm standard deviation for the atlas population.

Figure 4: ROC analysis against volume response of baseline motion abnormalities (systolic, at basal- and midinferoseptal levels) and conventional indexes of dyssynchrony (TDI septal-to-lateral and speckle tracking anteroseptal-to-inferolateral delays).

Figure 5: Average abnormality map for the set of volume responders and non-responders, at baseline and follow-up. 


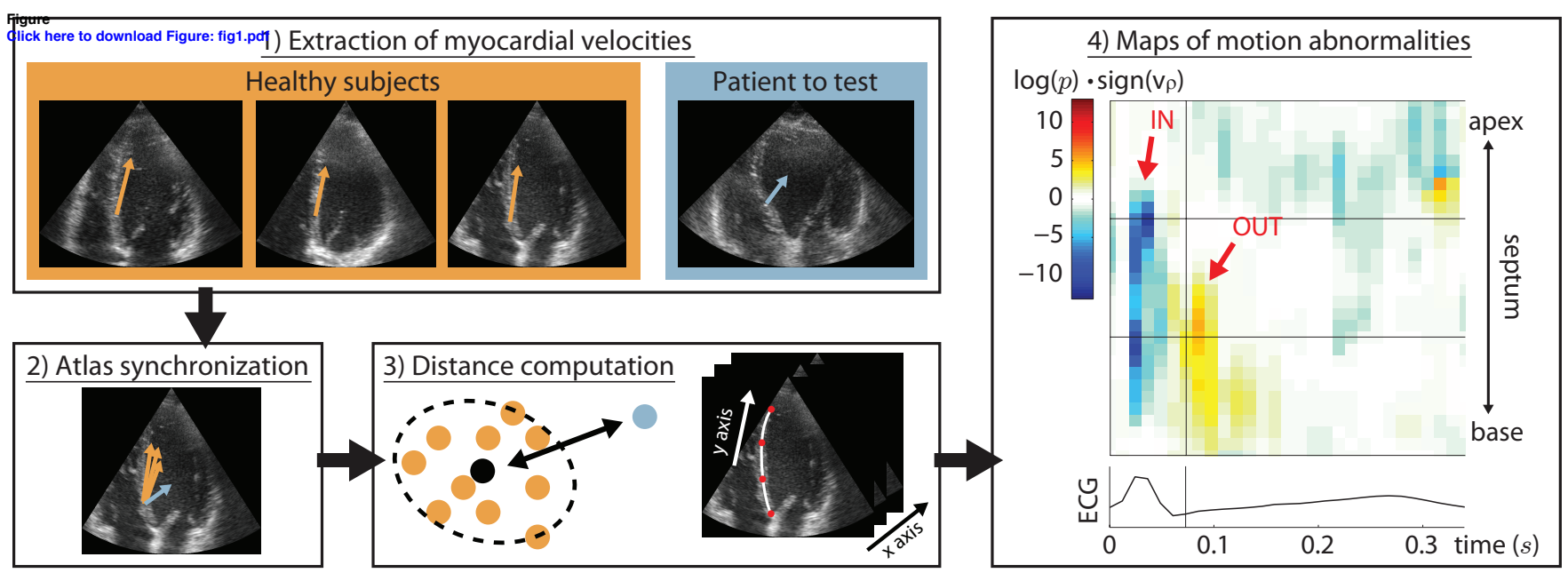


Preprint version accepted to appear in Ultrasound in Medicine and Biology.

Final version of this paper available at http://www.umbjournal.org/article/S0301-5629(12)00473-5/abstract
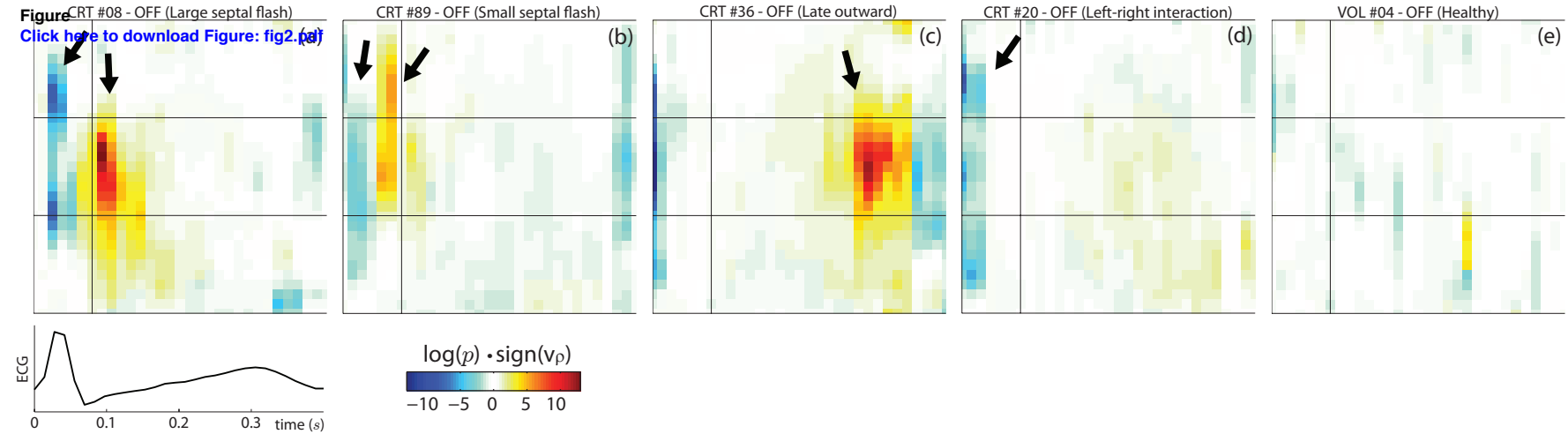

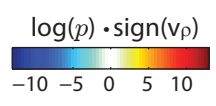


Cel $\mathbb{R}$ ak $\$$ bere to download Figure: fig3.pdf
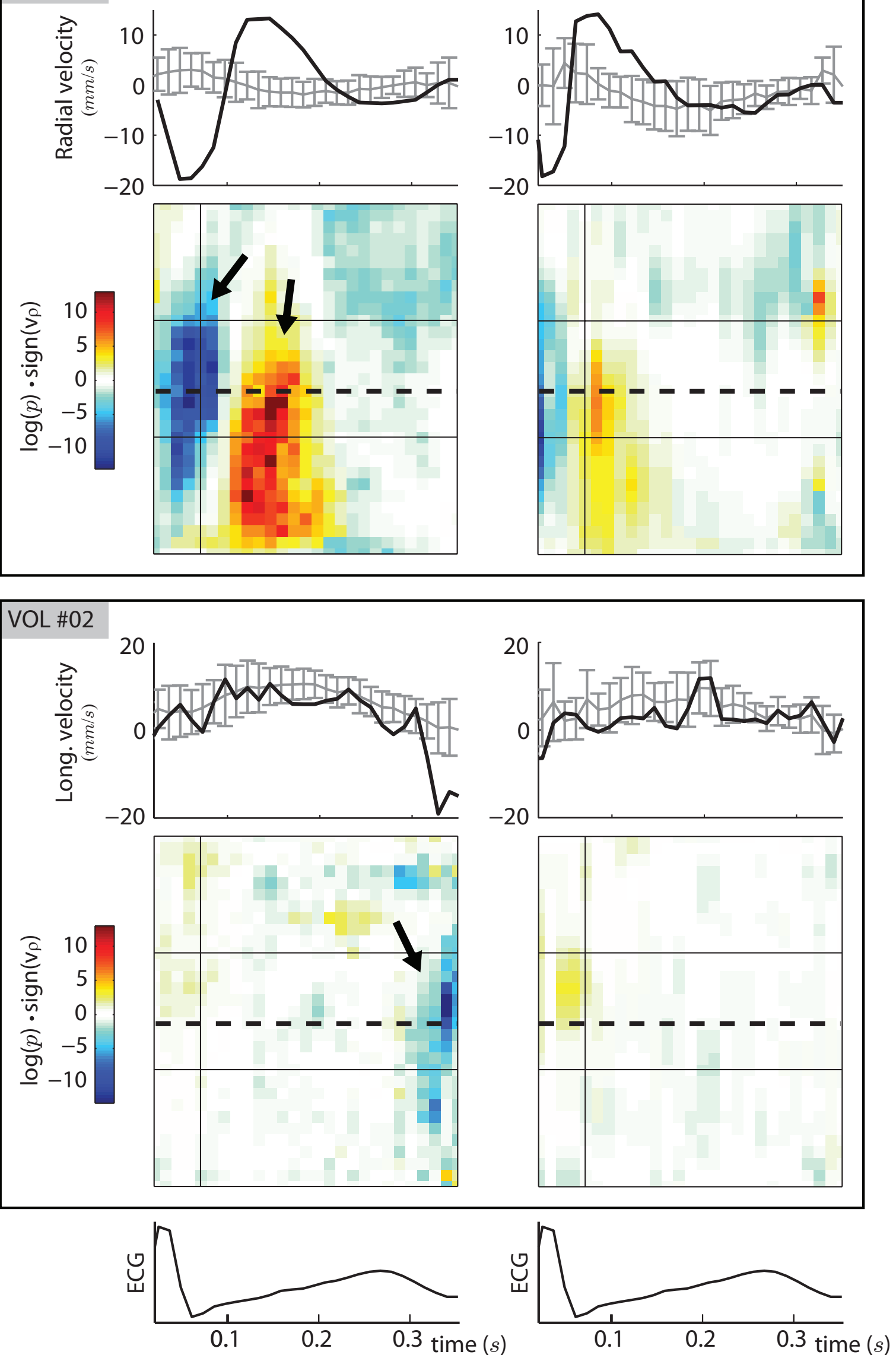


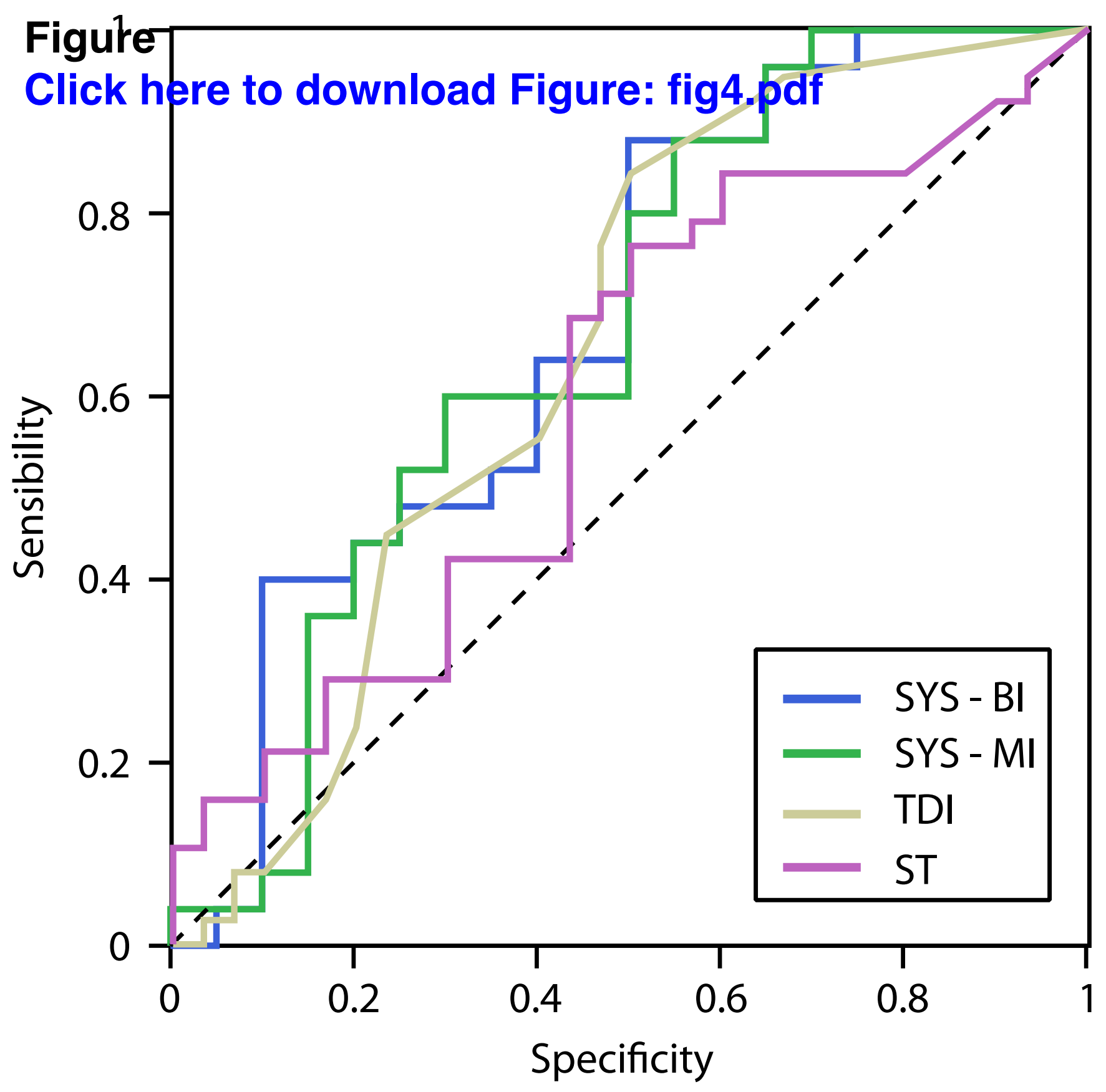

SYS - BI: systolic abnormality at basal-inferoseptal level SYS - MI: systolic abnormality at mid-inferoseptal level TDI: tissue Doppler imaging septal-to-lateral delay ST: speckle tracking anteroseptal-to-inferolateral delay 
Preprint version accepted to appear in Ultrasound in Medicine and Biology.

Final version of this paper available at http://www.umbjournal.org/article/S0301-5629(12)00473-5/abstract

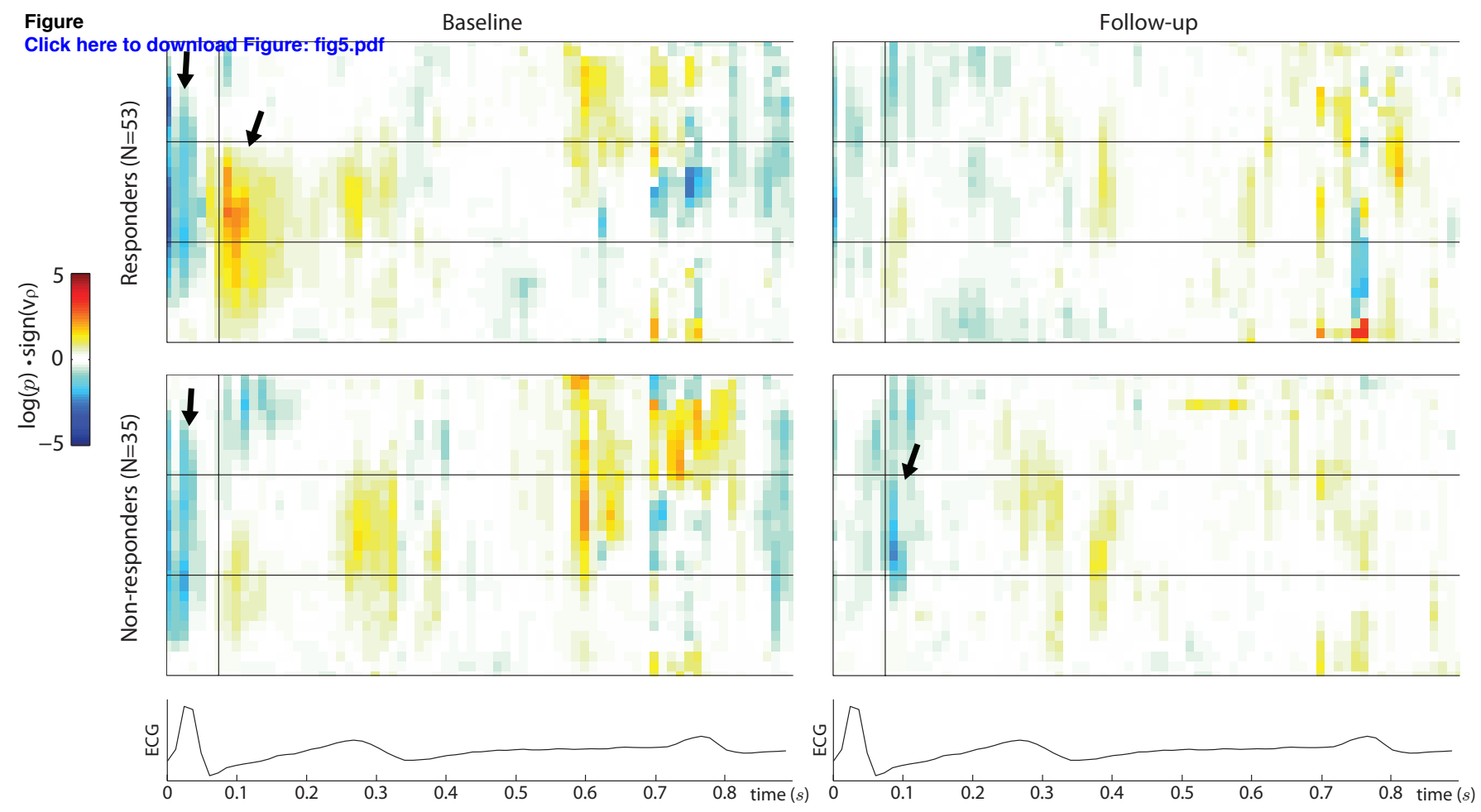

